\title{
CHEMICAL CONSTITUENTS FROM THE FRUIT OF CITRUS UNSHIU AND THEIR INHIBITORY EFFECTS ON ACETYLCHOLINESTERASE
}

\author{
Jiwon Baek ${ }^{1}$, Hye Lim Lee ${ }^{2}$, Ki Sung Kang ${ }^{2 *}$ Ki Hyun Kim ${ }^{1 *}$ \\ ${ }^{1}$ School of Pharmacy, Sungkyunkwan University, Suwon 16419, Republic of Korea \\ ${ }^{2}$ College of Korean Medicine, Gachon University, Seongnam 461-701, Republic of Korea \\ kkang@gachon.ac.kr, khkim83@skku.edu
}

\begin{abstract}
Acetylcholinesterase (AChE) inhibition has been considered a promising temporary treatment for Alzheimer's disease. The present study was conducted to isolate chemical constituents from the fruit of Citrus unshiu Markovich (Rutaceae) and evaluate their inhibitory effects on AChE. Phytochemical investigation of $C$. unshiu fruit led to the isolation of methyl nomilinate (1), limocitrunshin (2), nobiletin (3), kaempferol 3-O-rutinoside (4), limocitrin 3-glucoside (5), kaempferol 3-(2 ${ }^{G}$-rhamnosylrutinoside) (6), didymin (7), (2S)-narirutin 4'-O-glucoside (8) and naringenin 7-O-rutinoside (9). The structures were identified with ${ }^{1} \mathrm{H}$ and ${ }^{13} \mathrm{C}$ NMR spectroscopic data and ESI-MS, then comparison of their spectroscopic and physical data with those previously reported, as well as by measurement of their specific rotations. A limonoid, methyl nomilinate (1), inhibited AChE activity by more than $50 \%$ at a concentration of 30 $\mu \mathrm{g} / \mathrm{ml}$. The findings in this study provide the first experimental evidence that a limonoid compound $\mathbf{1}$ from $C$. unshiu fruit acts as a natural $\mathrm{AChE}$ inhibitor.
\end{abstract}

Keywords: Citrus unshiu; Rutaceae; limonoid; acetylcholinesterase

\section{ХЕМИСКИ СОСТАВ НА СОСТОЈКИТЕ ОД ПЛОДОТ НА CITRUS UNSHIU И НИВНОТО ИНХИБИТОРНО ДЕЈСТВО ВРЗ АЦЕТИЛХОЛИНЕСТЕРАЗАТА}

Инхибицијата на ацетилхолинестеразата (AChE) претставува потенцјален привремен третман за Алцхајмеровата болест. Цел на ова истражување беше да се изолираат хемиските состојки на Citrus unshiu Markovich (Rutaceae) и да се евалуира нивното инхибиторно дејство на AChE. При фитохемиското истражување на плодот на C. unshiu беа изолирани метил номилинат (1), лимоцинтрушин (2), нобилетин (3), кампферол 3-O-рутинозид (4), лимоцитрин 3-глукозид (5), кампферол 3-(2 ${ }^{G}$-рамнозилрутинозид) (6), дидимин (7), (2S)-нарирутин 4'-O-глукозид (8) and нарингенин 7-O-рутинозид (9). Структурите беа идентификувани преку податоци добиени од ${ }^{1} \mathrm{H}$ и ${ }^{13} \mathrm{C}$ NMR спектроскопија и ESI-MS, кои беа споредени со претходно објавени податоци, како и со мерење на аголот на нивните специфични ротации. Лимоноидот метил номилинат (1) ја инхибираше активноста на AChE за повеќе од 50\% при концентрација од $30 \mu \mathrm{g} / \mathrm{ml}$. Резултатите од овие испитувања се прв експериментален доказ дека лимоноидно соединение 1 од плодот на $C$. unshiu дејствува како природен инхибитор на AChE.

Клучни зборови: Citrus unshiu; Rutaceae; лимоноид; ацетилхолинестераза

\section{INTRODUCTION}

Alzheimer's disease (AD) is a chronic, neurodegenerative disorder characterized by progressive dementia $[1,2]$. AD will become a serious problem in an aging society because it usually occurs in the elderly. Therefore, research into its causes and practical treatments is becoming necessary. Many factors are involved in AD including aberrant protein processing, genetic effects, oxida- 
tive stress, cholinergic deficiency, inflammation and excitotoxicity. However, definitive AD therapies and diagnosis remain uncertain [3, 4]. One of the most promising approaches for $\mathrm{AD}$ treatment is acetylcholinesterase (AChE) inhibition, which can reduce acetylcholine levels in the brain [5]. There are only a few drugs that are approved by the Food and Drug Administration (FDA) for AChE inhibition, including tacrine, donepezil, rivastigmine and galantamine [6]. Thus, the search for new AChE inhibitors is highly necessary to broaden the selection of drugs available to treat $\mathrm{AD}$. The modern pharmaceutical field has focused its attention on natural products that have been used for centuries throughout the world. Thus, research on discovering new $\mathrm{AChE}$ inhibitors has been focused on compounds derived from natural sources.

Citrus unshiu Markovich (Rutaceae), also known as the satsuma mandarin, is cultivated in countries in cool subtropical regions including Korea, and is consumed in a variety of forms such as fresh juice, citrus-based drinks, jam, salad dressing and tea [7, 8]. Due to its sweet and fresh flavor, it is one of the most popular fruits in Korea. In addition, for centuries it has been consumed not only as food but also as a medicine. The peel of $C$. unshiu, well-known as "Jin-pee", has been used for traditional herbal medicines against the common cold, exhaustion and cancer [7, 9]. Previous studies have demonstrated that the peel has antitumor properties in a tumor-bearing murine model [9] as well as antioxidant activity [10-12]. The peel contains compounds associated with antioxidant and free radical scavenging effects such as ascorbic acid, flavonoids and phenolic compounds. In particular, phenolic compounds from the peel including flavonoids and hydroxyl cinnamic acids have a number of biological and pharmacological effects including anti-inflammatory, antimicrobial, antithrombotic, antiallergenic, cardioprotective and vasodilatory effects, along with hypocholesterolemic, hypoglycemic and antitumor activity [11, 12].

In our ongoing search for bioactive constituents from Korean natural resources, we have investigated bioactive compounds from the fruit of $C$. unshiu. In our recent studies, we reported the isolation of a novel cyclic peptide, citrusin XI, and its anti-inflammatory effects in lipopolysaccharide (LPS)-stimulated RAW 264.7 cells [13]. In addition, we discovered a new flavonoid glycoside, limocitrunshin, together with other flavonoids, and evaluated their inhibitory effects on renal cell damage and nitric oxide (NO) production [7]. NO is an important pro-inflammatory mediator that can accelerate the appearance of some neurodegenerative disorders such as AD [14]. Thus, the fruit of C. unshiu containing compounds with NO inhibitory properties may be a promising candidate for providing therapeutic agents for $\mathrm{AD}$. In our continued interest in discovering bioactive compounds of $C$. unshiu fruit, we further investigated such for possible new natural AChE inhibitors from the fruit of C. unshiu. Nine compounds designated as 1-9 including a limonoid and eight flavonoids were isolated, and the inhibitory effects of the isolates on AChE activity were evaluated using a micro-well plate AChE inhibition assay.

\section{EXPERIMENTAL}

\subsection{General experimental procedures}

Optical rotations were measured on a Jasco P-1020 polarimeter (Jasco, Easton, MD, USA). Infrared (IR) spectra were recorded on a Bruker IFS-66/S FT-IR spectrometer (Bruker, Karlsruhe, Germany). Ultraviolet (UV) spectra were acquired on an Agilent 8453 UV-visible spectrophotometer (Agilent Technologies, Santa Clara, CA, USA). High-resolution (HR)-electrospray ionization (ESI) mass spectra were recorded on a Waters UPLCQTOF Xevo G2-S mass spectrometer (Waters Corporation, Milford, CT, USA). Nuclear magnetic resonance (NMR) spectra were recorded on a Bruker AVANCE III 700 NMR spectrometer operating at $700 \mathrm{MHz}\left({ }^{1} \mathrm{H}\right)$ and $175 \mathrm{MHz}\left({ }^{13} \mathrm{C}\right)$, with chemical shifts given in ppm $(\delta)$ (Bruker). Preparative high-performance liquid chromatography (HPLC) purification was carried out on an Agilent 1100 or 1200 Series HPLC system (Agilent Technologies) equipped with a photo diode array detector. LC-MS analysis was performed on an Agilent 1200 Series HPLC system equipped with a diode array detector and a 6130 Series ESI mass spectrometer using an analytical Kinetex ${ }^{\circledR} 5 \mu \mathrm{m} \mathrm{C18} 100$ $\AA$ Å column $(5 \mu \mathrm{m}, 2.1 \times 100 \mathrm{~mm}$, Phenomenex, Torrance, CA, USA). Column chromatography was performed with a silica gel 60 (Merck, Darmstadt, Germany; 230-400 mesh) and an RP-C ${ }_{18}$ silica gel (Merck, 230-400 mesh). The packing material for molecular sieve column chromatography was Sephadex LH-20 (Pharmacia, Uppsala, Sweden). Merck precoated silica gel $\mathrm{F}_{254}$ plates and reversephase (RP)-18 $\mathrm{F}_{254 \mathrm{~s}}$ plates (Merck) were used for thin-layer chromatography (TLC). Spots were detected on TLC under UV light or by heating after spraying with anisaldehyde-sulfuric acid. 


\subsection{Plant material}

Fully ripe fruit of the "Miyagawa-wase" variety of satsuma mandarin (C. unshiu Marc.) were obtained from the National Institutes of Horticultural and Herbal Science, Jeju, Korea, in August 2012. Plant material was identified by one of the authors (K. H. Kim). A voucher specimen (SKKUCU 2012-8) has been stored in the herbarium of the School of Pharmacy, Sungkyunkwan University, Suwon, Korea.

\subsection{Extraction and isolation}

The unpeeled fruit of $C$. unshiu $(1.0 \mathrm{~kg})$ was extracted twice with $100 \% \mathrm{EtOH}(2 \times 2$ days $)$ at room temperature. The resultant extract was then filtered and concentrated under vacuum pressure to yield crude extracts. EtOH extracts $(89.0 \mathrm{~g})$ were suspended in distilled water (10 liters) and successively partitioned with hexane, EtOAc and $n$ $\mathrm{BuOH}$, yielding residues weighing $230 \mathrm{mg}, 3.0 \mathrm{~g}$ and $15.0 \mathrm{~g}$, respectively. The EtOAc-soluble fraction $(3.0 \mathrm{~g})$ was subjected to fractionation with silica gel column chromatography using a gradient solvent system of $n$-hexane-EtOAc $(1: 1), \mathrm{CHCl}_{3}$ $\mathrm{MeOH}(50: 1,10: 1,5: 1,2: 1$ and $1: 1)$ and $100 \%$ $\mathrm{MeOH}$ to give thirty fractions (E1-E30) according to TLC analysis. Fraction E7 (260 mg) was applied to column chromatography on an $\mathrm{RP}-\mathrm{C}_{18}$ silica gel column using a solvent system of $\mathrm{MeOH}-\mathrm{H}_{2} \mathrm{O}$ (2:1) to yield 10 sub-fractions (E7a-E7j). Fraction E7b $(52 \mathrm{mg})$ was further purified by semipreparative RP HPLC $(250 \mathrm{~mm} \times 10 \mathrm{~mm}$ i.d., 10 $\mu \mathrm{m}$, Phenomenex Luna C18(2) column, flow rate $1.5 \mathrm{ml} / \mathrm{min}$ ) using a solvent system of $\mathrm{MeOH}-\mathrm{H}_{2} \mathrm{O}$ (2:1) to afford compounds $\mathbf{1}(8.0 \mathrm{mg}), \mathbf{3}(12.0 \mathrm{mg})$ and $\mathbf{5}(6.4 \mathrm{mg})$. The $n$-BuOH soluble fraction $(15.0$ g) was subjected to fractionation with HP-20 column chromatography using a gradient solvent system that increased $\mathrm{MeOH}$ in $\mathrm{H}_{2} \mathrm{O}$ from $0 \%$ to $100 \%$ to yield six fractions (B1-B6) according to TLC analysis. Fraction B2 (4.5 g) was further subjected to silica gel column chromatography using a solvent system of $\mathrm{CHCl}_{3}-\mathrm{MeOH}-\mathrm{H}_{2} \mathrm{O}(7: 3: 1)$ to obtain 32 sub-fractions (B2(1)-B2(32)). Fraction B2(11) (122 mg) was purified by preparative RP HPLC $(250 \mathrm{~mm} \times 20 \mathrm{~mm}$ i.d., $10 \mu \mathrm{m}$, YMC-Pack ODS-AM C18(2) column, flow rate $8.0 \mathrm{ml} / \mathrm{min}$ ) using a $40 \% \mathrm{MeOH}$ solvent system to obtain compound $9(38.0 \mathrm{mg})$. Fraction B2(31) $(277 \mathrm{mg})$ was further separated by preparative RP HPLC (250 $\mathrm{mm} \times 20 \mathrm{~mm}$ i.d., $10 \mu \mathrm{m}$, YMC-Pack ODS-AM C18(2) column, flow rate $10.0 \mathrm{ml} / \mathrm{min}$ ) using a $40 \% \mathrm{MeOH}$ solvent system to yield compound 6
(9.0 mg), along with subfraction B2(31)A (87.0 $\mathrm{mg}$ ), which was further purified by semipreparative RP HPLC $(250 \mathrm{~mm} \times 10 \mathrm{~mm}$ i.d., 10 $\mu \mathrm{m}$, Phenomenex Luna C18(2) column, flow rate $2.0 \mathrm{ml} / \mathrm{min}$ ) with a $30 \% \mathrm{MeOH}$ solvent system to furnish compound $\mathbf{8}(30.0 \mathrm{mg})$. Fraction B4 (2.8 g) was applied to a silica gel column using a gradient of $\mathrm{CHCl}_{3}-\mathrm{MeOH}-\mathrm{H}_{2} \mathrm{O}(8: 3: 1,6: 3: 1$ and $6: 4: 1)$ and $100 \% \mathrm{MeOH}$ to give 29 sub-fractions (B4(1)B4(29)). Fraction B4(9) (44 mg) was purified by semi-preparative RP HPLC ( $250 \mathrm{~mm} \times 10 \mathrm{~mm}$ i.d., $10 \mu \mathrm{m}$, Phenomenex Luna C18(2) column, flow rate $2.0 \mathrm{ml} / \mathrm{min}$ ) using a $40 \% \mathrm{MeOH}$ solvent system to afford compound $7(5.2 \mathrm{mg})$. Fraction B4(15) (168 mg) was further separated by semipreparative RP HPLC $(250 \mathrm{~mm} \times 10 \mathrm{~mm}$ i.d., 10 $\mu \mathrm{m}$, Phenomenex Luna C18(2) column, flow rate $2.0 \mathrm{ml} / \mathrm{min}$ ) with a $40 \% \mathrm{MeOH}$ solvent system to yield compound $2(21.5 \mathrm{mg})$. Finally, fraction B6 (3.2 g) was subjected to silica gel column chromatography using a gradient of $\mathrm{CHCl}_{3}-\mathrm{MeOH}-\mathrm{H}_{2} \mathrm{O}$ $(8: 3: 1)$ and $100 \% \mathrm{MeOH}$ to give 20 sub-fractions (B6(1)-B6(20)). Fraction B6(10) (383 mg) was purified by semi-preparative RP HPLC ( $250 \mathrm{~mm} \times$ $10 \mathrm{~mm}$ i.d., $10 \mu \mathrm{m}$, Phenomenex Luna C18(2) column, flow rate $3.0 \mathrm{ml} / \mathrm{min}$ ) using a $40 \% \mathrm{MeOH}$ solvent system to obtain compound $\mathbf{4}(6.0 \mathrm{mg})$.

\subsubsection{Methyl nomilinate (1)}

${ }^{1} \mathrm{H}$ NMR (400 MHz, acetone- $\left.d_{6}\right) \delta: 1.12(\mathrm{~s}$, $3 \mathrm{H}, \mathrm{H}-18), 1.17$ (s, 3H, H-25a), 1.21 (s, 3H, H25b), 1.22 (s, 3H, H-24), 1.27 (s, 3H, H-19), 2.10 (s, $\left.3 \mathrm{H}, \mathrm{CH}_{3} \mathrm{COO}\right), 2.53(\mathrm{dd}, 1 \mathrm{H}, J=5.0,14.0 \mathrm{~Hz}$, H-6a), 2.60 (dd, $1 \mathrm{H}, J=10.5,14.0 \mathrm{~Hz}, \mathrm{H}-2 \mathrm{a})$, 2.61 (br d, $1 \mathrm{H}, J=14.0 \mathrm{~Hz}, \mathrm{H}-6 \mathrm{~b}$ ), 2.72 (br d, $1 \mathrm{H}$, $J=14.0 \mathrm{~Hz}, \mathrm{H}-2 \mathrm{~b}), 3.57$ (OMe), 3.76 (s, 1H, H15), 5.23 (br d, $1 \mathrm{H}, J=10.5 \mathrm{~Hz}, \mathrm{H}-1), 5.66(\mathrm{~s}, 1 \mathrm{H}$, $\mathrm{H}-17$ ), 6.44 (br s, 1H, H-22), 7.41 (br s, $1 \mathrm{H}, \mathrm{H}-$ 23), 7.74 (br s, $1 \mathrm{H}, \mathrm{H}-21) ;{ }^{13} \mathrm{C}$ NMR $(100 \mathrm{MHz}$, acetone- $\left.d_{6}\right) \delta: 16.0(\mathrm{C}-19), 16.8(\mathrm{C}-18), 17.4(\mathrm{C}-$ 11), 20.7 ( $\left.\mathrm{CH}_{3} \mathrm{COO}\right), 20.8$ (C-24), 23.1 (C-25b), 31.9 (C-12), 33.4 (C-25a), 35.3 (C-6), 37.4 (C13), 38.9 (C-2), 44.2 (C-9), 45.7 (C-10), 51.2 (OMe), $51.8(\mathrm{C}-5), 52.5(\mathrm{C}-8), 52.8(\mathrm{C}-15), 65.7$ (C-14), 73.2 (C-4), 76.8 (C-1), 77.8 (C-17), 110.2 (C-22), 120.3 (C-20), 141.4 (C-23), 143.2 (C-21), 166.9 (C-16), 169.5 ( $\left.\mathrm{CH}_{3} \mathrm{COO}\right), 171.3(\mathrm{C}-3)$, 209.8 (C-7); ESI-MS m/z 547.2 [M+H] ${ }^{+}$.

\subsubsection{Limocitrunshin (2)}

${ }^{1} \mathrm{H}$ NMR (400 MHz, $\left.\mathrm{CD}_{3} \mathrm{OD}\right) \delta: 1.23(\mathrm{~s}, 3 \mathrm{H}$, H-6"'), 2.52 (m, 4H, H-2"', 4"'), 3.33 (m, 1H, H4"), 3.39 (m, 1H, H-3"), 3.50 (m, 1H, H-5"), 3.53 
(m, 1H, H-2"), 3.94 (s, 3H, 8-OMe), 3.98 (s, 3H, 3'-OMe), 4.14 (dd, $1 \mathrm{H}, J=3.0,11.0 \mathrm{~Hz}, \mathrm{H}-6{ }^{\prime \prime} \mathrm{b}$ ), 4.25 (dd, 1H, $J=1.0,11.0 \mathrm{~Hz}, \mathrm{H}-6$ "a), 5.36 (d, $1 \mathrm{H}, J=7.5 \mathrm{~Hz}, \mathrm{H}-1^{\prime \prime}$ ), 6.29 (br s, 1H, H-6), 6.94 (d, $1 \mathrm{H}, J=8.0 \mathrm{~Hz}, \mathrm{H}-5^{\prime}$ ), 7.73 (br d, $1 \mathrm{H}, J=8.0$ $\left.\mathrm{Hz}, \mathrm{H}-6^{\prime}\right), 7.97$ (br s, 1H, H-2'); ${ }^{13} \mathrm{C}$ NMR (100 $\left.\mathrm{MHz}, \mathrm{CD}_{3} \mathrm{OD}\right) \delta: 26.9$ (C-6"'), $45.1\left(\mathrm{C}-4^{\prime \prime \prime}\right), 45.2$ $\left(\mathrm{C}-2^{\prime \prime \prime}\right), 55.8\left(3^{\prime}-\mathrm{OMe}\right), 61.2(8-\mathrm{OMe}), 63.6(\mathrm{C}-$ 6"), 69.7 (C-3"'), $70.8\left(\mathrm{C}-4^{\prime \prime}\right), 74.9\left(\mathrm{C}-2^{\prime \prime}\right), 75.0$ $\left(\mathrm{C}-5^{\prime \prime}\right), 77.0\left(\mathrm{C}-3^{\prime \prime}\right), 99.3(\mathrm{C}-6), 103.0\left(\mathrm{C}-1^{\prime \prime}\right)$, 104.7 (C-10), $113.3\left(\mathrm{C}-2^{\prime}\right), 115.2\left(\mathrm{C}-5^{\prime}\right), 122.1$ $\left(\mathrm{C}-1^{\prime}\right), 123.1\left(\mathrm{C}-6^{\prime}\right), 128.2(\mathrm{C}-8), 134.4(\mathrm{C}-3)$, $147.5\left(\mathrm{C}-3^{\prime}\right), 149.4(\mathrm{C}-9), 150.1\left(\mathrm{C}-4^{\prime}\right), 157.1(\mathrm{C}-$ 5), $157.6(\mathrm{C}-2), 157.9(\mathrm{C}-7), 171.3\left(\mathrm{C}-1^{\prime \prime \prime}\right), 171.5$ $\left(\mathrm{C}-5^{\prime \prime \prime}\right), 178.5(\mathrm{C}-4)$; ESI-MS $m / z$ 653.2 [M+H] $]^{+}$.

\subsubsection{Nobiletin (3)}

${ }^{1} \mathrm{H}$ NMR (400 MHz, DMSO- $\left.d_{6}\right) \delta: 3.96$ (s, 3H, 8-OMe), 3.97 (s, 3H, 6-OMe), 3.98 (s, 3H, 4'$\mathrm{OMe}), 4.00$ (s, 3H, 3'-OMe), 4.03 (s, 3H, 5-OMe), $4.11(\mathrm{~s}, 3 \mathrm{H}, 7-\mathrm{OMe}), 6.73(\mathrm{~s}, 1 \mathrm{H}, \mathrm{H}-3), 7.00(\mathrm{~d}$, $\left.1 \mathrm{H}, J=9.0 \mathrm{~Hz}, \mathrm{H}-5^{\prime}\right), 7.42(\mathrm{~d}, 1 \mathrm{H}, J=2.0 \mathrm{~Hz}, \mathrm{H}-$ $\left.2^{\prime}\right), 7.59\left(\mathrm{dd}, 1 \mathrm{H}, J=2.0,9.0 \mathrm{~Hz}, \mathrm{H}-6{ }^{\prime}\right) ;{ }^{13} \mathrm{C} \mathrm{NMR}$ $\left(100 \mathrm{MHz}, \mathrm{DMSO}-d_{6}\right) \delta: 55.6$ (6-OMe), 55.7 (8OMe), 61.4 (4'-OMe), 61.5 (3'-OMe), 61.8 (7OMe), 61.9 (5-OMe), 106.3 (C-3), 108.9 (C-2'), $111.9\left(\mathrm{C}-5^{\prime}\right), 114.3(\mathrm{C}-10), 119.3\left(\mathrm{C}-6^{\prime}\right), 123.1$ $\left(\mathrm{C}-1^{\prime}\right), 137.6(\mathrm{C}-6), 143.5(\mathrm{C}-5), 147.1$ (C-9), $147.5(\mathrm{C}-8), 149.0\left(\mathrm{C}-3^{\prime}\right), 150.9$ (C-7), 151.8 (C4'), 160.2 (C-2), 175.8 (C-4); ESI-MS $m / z 403.1$ $[\mathrm{M}+\mathrm{H}]^{+}$.

\subsubsection{Kaempferol 3-O-rutinoside (4)}

${ }^{1} \mathrm{H}$ NMR (400 MHz, $\left.\mathrm{CD}_{3} \mathrm{OD}\right) \delta: 1.11(\mathrm{~d}, 3 \mathrm{H}$, $\left.J=6.0 \mathrm{~Hz}, \mathrm{H}-6^{\prime \prime \prime}\right), 3.24\left(\mathrm{t}, 1 \mathrm{H}, J=9.0 \mathrm{~Hz}, \mathrm{H}-4^{\prime \prime}\right)$, 3.27 (t, $\left.1 \mathrm{H}, J=9.5 \mathrm{~Hz}, \mathrm{H}-4^{\prime \prime \prime}\right), 3.32$ (ddd, $1 \mathrm{H}, J=$ $\left.1.0,6.0,9.0 \mathrm{~Hz}, \mathrm{H}-5^{\prime \prime}\right), 3.37$ (dd, $1 \mathrm{H}, J=6.0,12.5$ $\mathrm{Hz}, \mathrm{H}-6^{\prime \prime} \mathrm{b}$ ), 3.40 (t, 1H, $\left.J=9.0 \mathrm{~Hz}, \mathrm{H}-3^{\prime \prime}\right), 3.43$ $\left(\mathrm{dd}, 1 \mathrm{H}, J=7.5,9.0 \mathrm{~Hz}, \mathrm{H}-2^{\prime \prime}\right), 3.44$ (dq, $1 \mathrm{H}, J=$ 6.0, $\left.9.5 \mathrm{~Hz}, \mathrm{H}-5^{\prime \prime \prime}\right), 3.51$ (dd, $1 \mathrm{H}, J=3.0,9.5 \mathrm{~Hz}$, H-3"'), 3.62 (dd, $\left.1 \mathrm{H}, J=1.5,3.0 \mathrm{~Hz}, \mathrm{H}-2^{\prime \prime \prime}\right), 3.80$ (dd, $1 \mathrm{H}, J=1.0,12.5 \mathrm{~Hz}, \mathrm{H}-6$ "a), 4.51 (d, $1 \mathrm{H}, J=$ $\left.1.5 \mathrm{~Hz}, \mathrm{H}-1^{\prime \prime \prime}\right), 5.12$ (d, $\left.1 \mathrm{H}, J=7.5 \mathrm{~Hz}, \mathrm{H}-1^{\prime \prime}\right)$, $6.21(\mathrm{~d}, 1 \mathrm{H}, J=2.0 \mathrm{~Hz}, \mathrm{H}-6), 6.40(\mathrm{~d}, 1 \mathrm{H}, J=2.0$ $\mathrm{Hz}, \mathrm{H}-8), 6.89$ (d, 2H, $\left.J=9.0 \mathrm{~Hz}, \mathrm{H}-3^{\prime}, 5^{\prime}\right), 8.05$ $\left(\mathrm{d}, 2 \mathrm{H}, J=9.0 \mathrm{~Hz}, \mathrm{H}-2^{\prime}, 6^{\prime}\right) ;{ }^{13} \mathrm{C}$ NMR $(100 \mathrm{MHz}$, $\left.\mathrm{CD}_{3} \mathrm{OD}\right) \delta: 17.9\left(\mathrm{C}-6^{\prime \prime \prime}\right), 68.6\left(\mathrm{C}-6^{\prime \prime}\right), 69.7(\mathrm{C}-$ 5"'), 71.5 (C-4"), $72.1\left(\mathrm{C}-2^{\prime \prime \prime}\right), 72.3\left(\mathrm{C}-3^{\prime \prime \prime}\right), 73.9$ $\left(\mathrm{C}-4^{\prime \prime \prime}\right), 75.8\left(\mathrm{C}-2^{\prime \prime}\right), 77.2\left(\mathrm{C}-5^{\prime \prime}\right), 78.2\left(\mathrm{C}-3^{\prime \prime}\right)$, 95.0 (C-8), 99.9 (C-6), $102.4\left(\mathrm{C}-1^{\prime \prime \prime}\right), 104.6(\mathrm{C}-$ $\left.1^{\prime \prime}\right), 105.7(\mathrm{C}-10), 116.1\left(\mathrm{C}-3^{\prime}, 5^{\prime}\right), 122.8\left(\mathrm{C}-1^{\prime}\right)$, $132.4\left(\mathrm{C}-2^{\prime}, 6^{\prime}\right), 135.5$ (C-3), 158.6 (C-9), 159.4 (C-4'), 161.5 (C-2), $163.0(\mathrm{C}-5), 166.0(\mathrm{C}-7)$, 179.4 (C-4); ESI-MS $m / z 595.2[\mathrm{M}+\mathrm{H}]^{+}$.

\subsubsection{Limocitrin 3-glucoside (5)}

${ }^{1} \mathrm{H}$ NMR $\left(400 \mathrm{MHz}\right.$, DMSO- $\left.d_{6}\right) \delta: 3.08(\mathrm{~m}$, $\left.1 \mathrm{H}, \mathrm{H}-5^{\prime \prime}\right), 3.10-3.40\left(\mathrm{~m}, 1 \mathrm{H}, \mathrm{H}-3^{\prime \prime}\right), 3.40(\mathrm{~m}, 1 \mathrm{H}$, H-6"a), 3.57 (br d, 1H, $J=11.5 \mathrm{~Hz}, \mathrm{H}-6^{\prime \prime} \mathrm{b}$ ), 3.81 (s, 3H, 8-OMe), 3.90 (s, 3H, 3'-OMe), 5.46 (d, $\left.1 \mathrm{H}, J=7.0 \mathrm{~Hz}, \mathrm{H}-1^{\prime \prime}\right), 6.57(\mathrm{~s}, 1 \mathrm{H}, \mathrm{H}-6), 6.86(\mathrm{~d}$, $1 \mathrm{H}, J=8.0 \mathrm{~Hz}, \mathrm{H}-5^{\prime}$ ), 7.63 (br s, $1 \mathrm{H}, \mathrm{H}-2^{\prime}$ ), 7.65 (dd, $\left.1 \mathrm{H}, J=2.0,9.0 \mathrm{~Hz}, \mathrm{H}-6^{\prime}\right), 12.48$ (s, 1H, 5$\mathrm{OH}) ;{ }^{13} \mathrm{C}$ NMR (100 MHz, DMSO- $d_{6}$, $\delta$ : $56.5\left(3^{\prime}-\right.$ OMe), 60.9 (C-6"), 61.1 (8-OMe), $69.9\left(\mathrm{C}-4^{\prime \prime}\right)$, 74.0 (C-2"), $76.4\left(\mathrm{C}-3^{\prime \prime}\right), 77.5\left(\mathrm{C}-5^{\prime \prime}\right), 95.7(\mathrm{C}-6)$, $100.9\left(\mathrm{C}-1^{\prime \prime}\right), 104.2(\mathrm{C}-10), 115.2\left(\mathrm{C}-2^{\prime}\right), 116.0$ $\left(\mathrm{C}-5^{\prime}\right), 121.2\left(\mathrm{C}-1^{\prime}\right), 121.7\left(\mathrm{C}-6^{\prime}\right), 128.3(\mathrm{C}-8)$, $133.2(\mathrm{C}-3), 144.9\left(\mathrm{C}-3^{\prime}\right), 147.7\left(\mathrm{C}-4^{\prime}\right), 148.7(\mathrm{C}-$ 9), $156.3(\mathrm{C}-2,5), 158.0(\mathrm{C}-7), 177.7$ (C-4); ESIMS $m / z 509.1[\mathrm{M}+\mathrm{H}]^{+}$.

\subsubsection{Kaempferol 3-( $2^{G}$-rhamnosylrutinoside) (6)}

${ }^{1} \mathrm{H}$ NMR $\left(400 \mathrm{MHz}, \mathrm{CD}_{3} \mathrm{OD}\right) \delta: 0.98(\mathrm{~d}, 3 \mathrm{H}$, $\left.J=6.0 \mathrm{~Hz}, \mathrm{H}-6^{\prime \prime \prime}\right), 1.07$ (d, 3H, $J=6.0 \mathrm{~Hz}, \mathrm{H}-$ $\left.6^{\prime \prime \prime}\right), 3.23\left(\mathrm{t}, 1 \mathrm{H}, J=9.0 \mathrm{~Hz}, \mathrm{H}-4^{\prime \prime}\right), 3.23(\mathrm{t}, 1 \mathrm{H}, J$ $\left.=9.5 \mathrm{~Hz}, \mathrm{H}-4^{\prime \prime \prime}\right), 3.33$ (ddd, $1 \mathrm{H}, J=1.0,6.0,9.0$ $\left.\mathrm{Hz}, \mathrm{H}-5^{\prime \prime}\right), 3.33$ (t, 1H, $\left.J=9.5 \mathrm{~Hz}, \mathrm{H}-4^{\prime \prime \prime \prime}\right), 3.37$ (dd, $\left.1 \mathrm{H}, J=6.0,12.5 \mathrm{~Hz}, \mathrm{H}-6^{\prime \prime} \mathrm{b}\right), 3.41$ (dq, $1 \mathrm{H}, J$ $\left.=6.0,9.5 \mathrm{~Hz}, \mathrm{H}-5^{\prime \prime \prime}\right), 3.47$ (dd, $1 \mathrm{H}, J=3.5,9.5$ $\left.\mathrm{Hz}, \mathrm{H}-3^{\prime \prime \prime}\right), 3.53$ (t, 1H, $\left.J=9.0 \mathrm{~Hz}, \mathrm{H}-3^{\prime \prime}\right), 3.57$ (dd, $\left.1 \mathrm{H}, J=1.5,3.5 \mathrm{~Hz}, \mathrm{H}-2^{\prime \prime \prime}\right), 3.59$ (dd, $1 \mathrm{H}, J=$ 7.5, $\left.9.0 \mathrm{~Hz}, \mathrm{H}-2^{\prime \prime}\right), 3.78(\mathrm{dd}, 1 \mathrm{H}, J=3.5,9.5 \mathrm{~Hz}$, H-3'"'), 3.81 (dd, 1H, $J=1.0,12.5 \mathrm{~Hz}, \mathrm{H}-6^{\prime \prime} \mathrm{a}$ ), 3.99 (dd, $\left.1 \mathrm{H}, J=1.5,3.5 \mathrm{~Hz}, \mathrm{H}-2^{\prime \prime \prime \prime}\right), 4.05$ (dq, $\left.1 \mathrm{H}, J=6.0,9.5 \mathrm{~Hz}, \mathrm{H}-5^{\prime \prime \prime \prime}\right), 4.49$ (d, $1 \mathrm{H}, J=1.5$ $\left.\mathrm{Hz}, \mathrm{H}-1^{\prime \prime \prime}\right), 5.22$ (d, $\left.1 \mathrm{H}, J=1.5 \mathrm{~Hz}, \mathrm{H}-1^{\prime \prime \prime \prime}\right), 5.59$ $\left(\mathrm{d}, 1 \mathrm{H}, J=7.5 \mathrm{~Hz}, \mathrm{H}-1^{\prime \prime}\right), 6.18(\mathrm{~d}, 1 \mathrm{H}, J=2.0 \mathrm{~Hz}$, H-6), 6.37 (d, 1H, $J=2.0 \mathrm{~Hz}, \mathrm{H}-8), 6.89$ (d, 2H, $J$ $\left.=9.0 \mathrm{~Hz}, \mathrm{H}-3^{\prime}, 5^{\prime}\right), 8.01\left(\mathrm{~d}, 2 \mathrm{H}, J=9.0 \mathrm{~Hz}, \mathrm{H}-2^{\prime}\right.$, $\left.6^{\prime}\right) ;{ }^{13} \mathrm{C}$ NMR (100 MHz, $\left.\mathrm{CD}_{3} \mathrm{OD}\right) \delta: 17.6\left(\mathrm{C}-6^{\prime \prime \prime \prime}\right)$, $17.8\left(\mathrm{C}-6^{\prime \prime \prime}\right), 68.3\left(\mathrm{C}-6^{\prime \prime}\right), 69.8\left(\mathrm{C}-5^{\prime \prime \prime}\right), 69.9(\mathrm{C}-$ $\left.5^{\prime \prime \prime \prime}\right), 72.0$ (C-4"), 72.1 (C-2'"'), 72.3 (C-3'"', 3"''), $72.4\left(\mathrm{C}-2^{\prime \prime \prime \prime}\right), 73.8\left(\mathrm{C}-4^{\prime \prime \prime}\right), 74.1\left(\mathrm{C}-4^{\prime \prime \prime \prime}\right), 77.1(\mathrm{C}-$ 5"), 78.9 (C-3"), 79.9 (C-2"), 94.8 (C-8), 99.8 (C-6), $100.5\left(\mathrm{C}-1^{\prime \prime}\right), 102.3\left(\mathrm{C}-1^{\prime \prime \prime}\right), 102.6\left(\mathrm{C}-1^{\prime \prime \prime \prime}\right)$, 105.9 (C-10), $116.1\left(\mathrm{C}-3^{\prime}, 5^{\prime}\right), 123.2\left(\mathrm{C}-1^{\prime}\right), 132.1$ (C-2', 6'), $134.3(\mathrm{C}-3), 158.5(\mathrm{C}-9), 159.0\left(\mathrm{C}-4^{\prime}\right)$, 161.2 (C-2), 163.2 (C-5), 165.6 (C-7), 179.3 (C4); ESI-MS $m / z 741.2[\mathrm{M}+\mathrm{H}]^{+}$.

\subsubsection{Didymin (7)}

${ }^{1} \mathrm{H}$ NMR $\left(400 \mathrm{MHz}\right.$, DMSO- $\left.d_{6}\right) \delta: 1.07$ (d, $\left.3 \mathrm{H}, J=6.2 \mathrm{~Hz}, \mathrm{H}-6^{\prime \prime \prime}\right), 2.77$ (dd, $1 \mathrm{H}, J=2.5,17.0$ Hz, H-3a), 3.77 (s, 3H, 4'-OMe), 4.52 (br s, 1H, $\left.\mathrm{H}-1^{\prime \prime \prime}\right), 4.98$ (d, 1H, $\left.J=7.5 \mathrm{~Hz}, \mathrm{H}-1^{\prime \prime}\right), 5.58$ (dd, $1 \mathrm{H}, J=3.0,12.5 \mathrm{~Hz}, \mathrm{H}-2), 6.13$ (s, 2H, H-6, 8), $6.98\left(\mathrm{~d}, 2 \mathrm{H}, J=8.0 \mathrm{~Hz}, \mathrm{H}-3^{\prime}, 5^{\prime}\right), 7.47(\mathrm{~d}, 2 \mathrm{H}, J=$ 
$\left.8.5 \mathrm{~Hz}, \mathrm{H}-2^{\prime}, 6^{\prime}\right), 12.02$ (s, 1H, 5-OH); ${ }^{13} \mathrm{C} \mathrm{NMR}$ $\left(100 \mathrm{MHz}, \mathrm{DMSO}-d_{6}\right) \delta: 18.0\left(\mathrm{C}-6^{\prime \prime \prime}\right), 42.0(\mathrm{C}-3)$, 55.3 (4'-OMe), $66.1\left(\mathrm{C}-6^{\prime \prime}\right), 68.5\left(\mathrm{C}-5^{\prime \prime \prime}\right), 69.8(\mathrm{C}-$ $\left.4^{\prime \prime}\right), 70.4\left(\mathrm{C}-3^{\prime \prime \prime}\right), 70.8\left(\mathrm{C}-2^{\prime \prime \prime}\right), 72.2\left(\mathrm{C}-4^{\prime \prime \prime}\right), 73.1$ $\left(\mathrm{C}-2^{\prime \prime}\right), 75.7\left(\mathrm{C}-3^{\prime \prime}\right), 76.4\left(\mathrm{C}-5^{\prime \prime}\right), 78.5(\mathrm{C}-2), 95.6$ (C-8), $96.6(\mathrm{C}-6), 99.5\left(\mathrm{C}-1^{\prime \prime \prime}\right), 100.8\left(\mathrm{C}-1^{\prime \prime}\right)$, $103.4(\mathrm{C}-10), 114.1\left(\mathrm{C}-3^{\prime}, 5^{\prime}\right), 128.5\left(\mathrm{C}-6^{\prime}\right), 128.7$ $\left(\mathrm{C}-2^{\prime}\right), 130.5\left(\mathrm{C}-1^{\prime}\right), 159.7\left(\mathrm{C}-4^{\prime}\right), 162.7(\mathrm{C}-9)$, $163.2(\mathrm{C}-5), 165.3(\mathrm{C}-7), 197.2(\mathrm{C}-4)$; ESI-MS $\mathrm{m} / \mathrm{z} 595.2[\mathrm{M}+\mathrm{H}]^{+}$.

\subsection{8. (2S)-Narirutin 4'-O-glucoside (8)}

${ }^{1} \mathrm{H}$ NMR $\left(400 \mathrm{MHz}, \mathrm{CD}_{3} \mathrm{OD}\right) \delta: 1.21(\mathrm{~d}, 3 \mathrm{H}$, $\left.J=6.0 \mathrm{~Hz}, \mathrm{H}-6^{\prime \prime \prime}\right), 2.81(\mathrm{dd}, 1 \mathrm{H}, J=3.0,17.0 \mathrm{~Hz}$, $\mathrm{H}-3), 4.71$ (d, 1H, $\left.J=1.0 \mathrm{~Hz}, \mathrm{H}-1^{\prime \prime \prime}\right), 5.49$ (dd, $1 \mathrm{H}, J=3.0,12.0 \mathrm{~Hz}, \mathrm{H}-2), 6.22$ (s, 2H, H-6, 8), $7.17\left(\mathrm{~d}, 2 \mathrm{H}, J=8.0 \mathrm{~Hz}, \mathrm{H}-3^{\prime}, 5^{\prime}\right), 7.46$ (d, 2H, $J$ $\left.=8.0 \mathrm{~Hz}, \mathrm{H}-2^{\prime}, 6^{\prime}\right), 11.70(\mathrm{~s}, 1 \mathrm{H}, 5-\mathrm{OH}) ;{ }^{13} \mathrm{C}-$ NMR (100 MHz, CD 3 OD) $\delta: 18.1\left(\mathrm{C}-6^{\prime \prime \prime}\right), 43.5$ (C-3), 62.3 (C-6"'"'), $67.6\left(\mathrm{C}-6^{\prime \prime}\right), 69.9\left(\mathrm{C}-5^{\prime \prime \prime}\right)$, 71.5 (C-4"', 4'"'), 72.2 (C-2'"'), 72.5 (C-3'"'), 74.2 $\left(\mathrm{C}-4^{\prime \prime \prime}\right), 74.8\left(\mathrm{C}-2^{\prime \prime}\right), 75.0\left(\mathrm{C}-2^{\prime \prime \prime \prime}\right), 77.3\left(\mathrm{C}-5^{\prime \prime}\right)$, $78.0\left(\mathrm{C}-3^{\prime \prime}\right), 78.1\left(\mathrm{C}-5^{\prime \prime \prime \prime}\right), 78.3\left(\mathrm{C}-3^{\prime \prime \prime \prime}\right), 80.3(\mathrm{C}-$ 2), 97.3 (C-8), 98.2 (C-6), $101.3\left(\mathrm{C}-1^{\prime \prime \prime}\right), 102.3$ (C-1", 1'"'), 105.1 (C-10), $118.0\left(\mathrm{C}-3^{\prime}, 5^{\prime}\right), 129.1$ $\left(\mathrm{C}-2^{\prime}, 6^{\prime}\right), 134.06\left(\mathrm{C}-1^{\prime}\right), 159.4\left(\mathrm{C}-4^{\prime}\right), 164.5(\mathrm{C}-$ 9), $165.1(\mathrm{C}-5), 167.0(\mathrm{C}-7), 198.4$ (C-4); ESIMS $m / z 743.2[\mathrm{M}+\mathrm{H}]^{+}$.

\subsubsection{Naringenin 7-O-rutinoside (9)}

${ }^{1} \mathrm{H}$ NMR $\left(400 \mathrm{MHz}, \mathrm{CD}_{3} \mathrm{OD}\right) \delta: 2.68(\mathrm{dd}$, $1 \mathrm{H}, J=3.0,17.0 \mathrm{~Hz}, \mathrm{H}-3 \mathrm{~b}), 3.09$ (dd, $1 \mathrm{H}, J=$ 12.0, 17.0 Hz, H-3a), 5.29 (dd, $1 \mathrm{H}, J=3.0,12.0$ $\mathrm{Hz}, \mathrm{H}-2), 6.14$ (d, 2H, $J=1.0 \mathrm{~Hz}, \mathrm{H}-6,8), 6.81$ (d, 2H, $\left.J=8.0 \mathrm{~Hz}, \mathrm{H}-3^{\prime}, 5^{\prime}\right), 7.27$ (d, 2H, $J=8.0$ $\left.\mathrm{Hz}, \mathrm{H}-2^{\prime}, 6^{\prime}\right) ;{ }^{13} \mathrm{C}-\mathrm{NMR}\left(100 \mathrm{MHz}, \mathrm{CD}_{3} \mathrm{OD}\right) \delta$ : $17.9\left(\mathrm{C}-6^{\prime \prime \prime}\right), 43.9$ (C-3), $67.4\left(\mathrm{C}-6^{\prime \prime}\right), 69.7(\mathrm{C}-$ 5"'), 71.2 (C-4"), 71.9 (C-2"'), 72.3 (C-3"'), 74.0 (C-4"'), $74.5\left(\mathrm{C}-2^{\prime \prime}\right), 77.0\left(\mathrm{C}-5^{\prime \prime}\right), 77.7\left(\mathrm{C}-3^{\prime \prime}\right)$, 80.4 (C-2), 97.2 (C-8), 98.0 (C-6), $101.0\left(\mathrm{C}-1^{\prime \prime}\right)$, $102.0\left(\mathrm{C}-1^{\prime \prime \prime}\right), 104.9(\mathrm{C}-10), 116.4\left(\mathrm{C}-3^{\prime}, 5^{\prime}\right)$, $129.0\left(\mathrm{C}-2^{\prime}, 6^{\prime}\right), 130.7\left(\mathrm{C}-1^{\prime}\right), 158.8$ (C-9), 164.3 (C-4'), 164.7 (C-5), 166.7 (C-7), 198.3 (C-4); ESI-MS $m / z 581.2[\mathrm{M}+\mathrm{H}]^{+}$.

\subsection{AChE inhibition activity}

An Acetylcholinesterase Activity Colorimetric Assay Kit was purchased from BioVision (Milpitas, CA, USA) and AChE inhibition activity was measured using the AChE assay kit according to the manufacturer's protocol. In brief, $50 \mu \mathrm{l}$ of sample $(30 \mu \mathrm{g} / \mathrm{ml})$ and assay buffer was added to each well in a 96-well plate, followed by the addition of $50 \mu \mathrm{l}$ of the reaction mixture. After incubation at $37{ }^{\circ} \mathrm{C}$ for $20 \mathrm{~min}$, absorbance at $570 \mathrm{~nm}$ was measured using a microplate reader (PowerWave XS; Bio-Tek Instruments, Winooski, VT, USA). Aricept $(10 \mu \mathrm{M})$ was used as a positive control.

\subsection{Statistical analysis}

Statistical significance was determined with analysis of variance (ANOVA) followed by a multiple comparison test with Bonferroni adjustment. $P$ values less than 0.05 were considered statistically significant. Analyses were performed using SPSS version 19.0 (SPSS Inc., Chicago, IL, USA).

\section{RESULTS AND DISCUSSION}

\subsection{Isolation and structural elucidation of compounds}

The unpeeled C. unshiu fruit was extracted with $100 \% \mathrm{EtOH}$ to give crude $\mathrm{EtOH}$ extracts $(89.0 \mathrm{~g})$ that were solvent-partitioned with hexane, EtOAc and $n$-BuOH. Phytochemical investigation of the EtOAc and $n$-BuOH soluble fractions using repeated column chromatography and HPLC purification resulted in isolation and identification of nine compounds, 1-9 (Fig. 1). The compounds were identified as methyl nomilinate (1) [15], limocitrunshin (2) [7], nobiletin (3) [16], kaempferol 3-O-rutinoside (4) [17], limocitrin 3-glucoside (5) [18], kaempferol 3-(2 ${ }^{G}$-rhamnosylrutinoside) (6) [17], didymin (7) [19], (2S)-narirutin 4'-Oglucoside (8) [20] and naringenin 7-O-rutinoside (9) [21], respectively, based on comparison of their spectroscopic and physical data with those previously reported as well as by measurement of their specific rotations.

\subsection{Evaluation of AChE inhibition activity of the isolates}

Compounds 1-9 were evaluated for inhibitory effects on $\mathrm{AChE}$ to verify their potential health benefits. The inhibition effects of compounds 1-9 on AChE activity were examined using a microwell plate AChE inhibition assay [22]. 


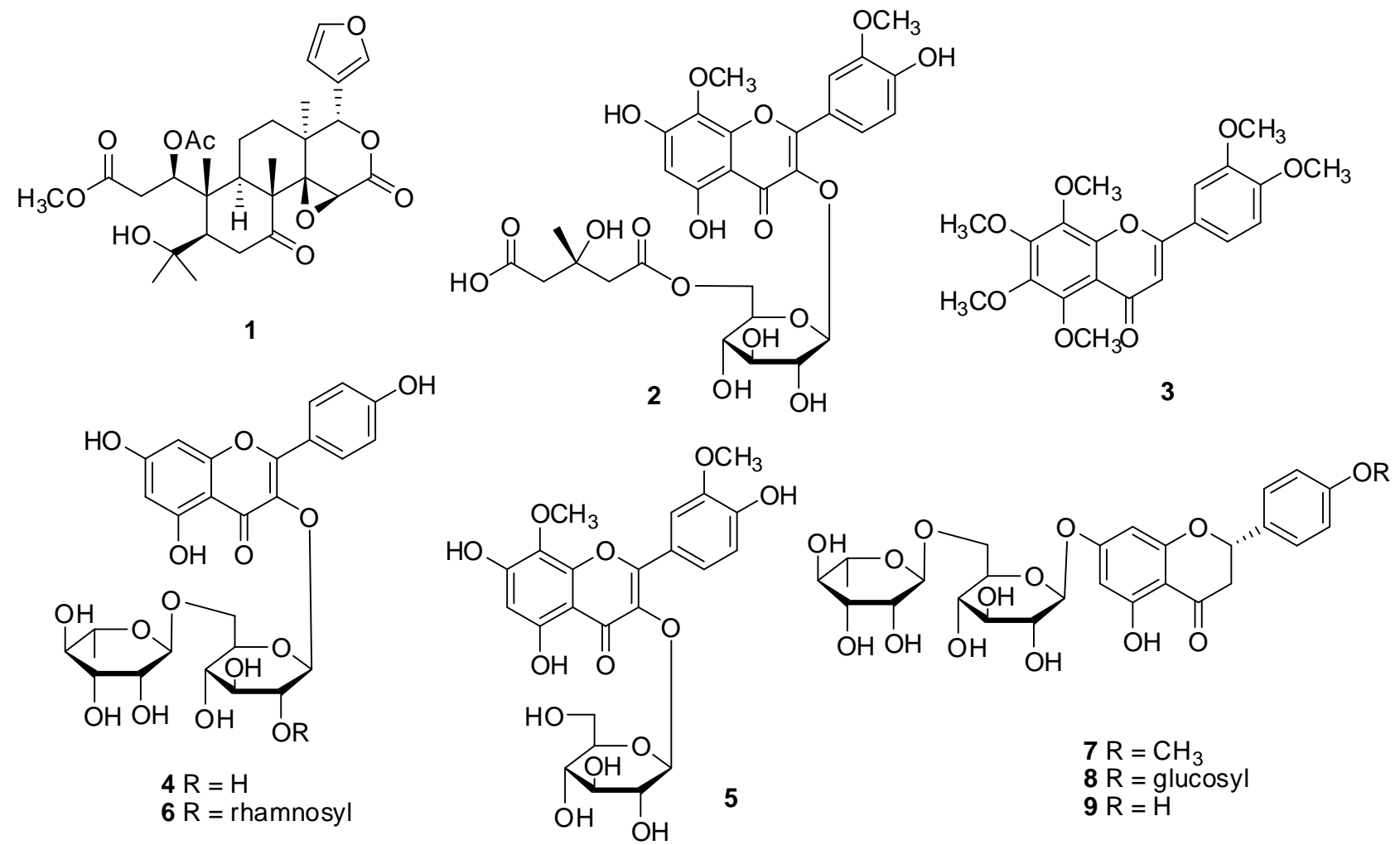

Fig. 1. Chemical structures of compounds 1-9

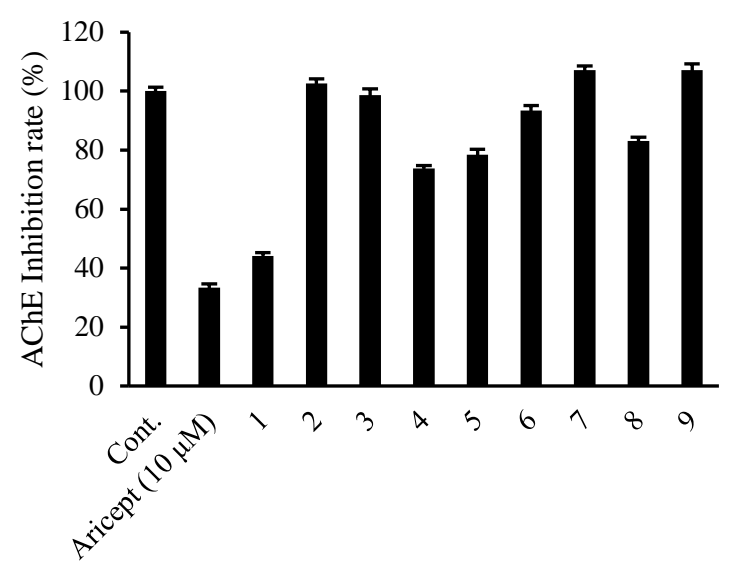

Fig. 2. Acetylcholinesterase inhibition rate (\%) comparison for compounds 1-9. Fifty $\mu 1$ of sample $(30 \mu \mathrm{g} / \mathrm{ml})$ and assay buffer was added to each well of a 96-well plate, followed by the addition of $50 \mu 1$ of the reaction mixture. After incubation at $37{ }^{\circ} \mathrm{C}$ for $20 \mathrm{~min}$, the absorbance at $570 \mathrm{~nm}$ was measured using a microplate reader. Aricept $(10 \mu \mathrm{M})$ was used as a positive control.

Inhibition of $\mathrm{AChE}$ activity leading to an increase in acetylcholine concentration in the brain is recognized as a potential treatment for mild to moderate $\mathrm{AD}$ and various other memory impairment disorders [23-27]. At a concentration of $30 \mu \mathrm{g} / \mathrm{ml}$, compound 1 inhibited $\mathrm{AChE}$ activity by more than $50 \%$ (Fig. 2). Compared with the control, com- pound 1 showed a $44.11 \pm 1.1 \% \mathrm{AChE}$ inhibition. This effect was similar to the $34.40 \pm 1.2 \%$ inhibition seen with a $10 \mu \mathrm{M}$ concentration of Aricept (positive control). However, the other compounds showed weak (compounds $\mathbf{4}, \mathbf{5}, \mathbf{6}$ and $\mathbf{8}$ ) to no inhibition (compounds 2, 3, 7 and 9) of AChE activity when compared to the control (Table 1).

Table 1

The acetylcholinesterase inhibition rate (\%) for compounds 1-9 compared to the control

\begin{tabular}{cc}
\hline \hline Compound & $\begin{array}{c}\text { AChE inhibition rate }(\%) \\
\text { at } 30 \mu \mathrm{g} / \mathrm{ml}\end{array}$ \\
\hline $\mathbf{1}$ & $44.11 \pm 1.1$ \\
$\mathbf{2}$ & $102.62 \pm 1.5$ \\
$\mathbf{3}$ & $98.69 \pm 2.1$ \\
$\mathbf{4}$ & $73.78 \pm 1.0$ \\
$\mathbf{5}$ & $78.42 \pm 1.9$ \\
$\mathbf{6}$ & $93.44 \pm 1.7$ \\
$\mathbf{7}$ & $107.04 \pm 1.5$ \\
$\mathbf{8}$ & $83.11 \pm 1.3$ \\
$\mathbf{9}$ & $107.11 \pm 2.1$ \\
Aricept $^{\mathrm{a}}$ & $33.40 \pm 1.2$ \\
\hline \hline
\end{tabular}

${ }^{a}$ Aricept was used as a positive control for $\mathrm{AChE}$ inhibition activity and its AChE inhibition rate (\%) was measured at a concentration of $10 \mu \mathrm{M}$. 


\subsection{Limonoid as a potential AChE activity inhibitor}

In the micro-well plate AChE inhibition assay of compounds $\mathbf{1}-\mathbf{9}$, compound $\mathbf{1}$ was the most potent AChE activity inhibitor. Compound $\mathbf{1}$ is a highly oxygenated limonoid compound with a prototypical structure consisting of four six-membered rings and a furan ring. This type of limonoid compound is abundant in citrus fruit and other plants from the families Rutaceae and Meliaceae [14]. Limonoids have been reported to exhibit diverse bioactivity including insecticidal, antibacterial, antifungal, antiviral and anticancer effects [28]. There have been widespread efforts to identify new AChE inhibitors from natural sources, but only limited work has focused on limonoids. Recently, three limonoids isolated from Trichilia welwitschii were reported to show weak to moderate inhibitory effects on AChE activity [14, 29]. Our present findings suggest for the first time that another limonoid, methyl nomilinate (1), is a potential AChE activity inhibitor.

In our previous study, we found that methyl nomilinate showed an anti-inflammatory effect by inhibiting NO production by LPS-activated RAW 264.7 macrophages and significantly abrogated cisplatin-induced nephrotoxicity in LLC-PK1 cells [7]. In addition, methyl nomilinate was reported to inhibit the proliferation of MCF-7 ER+ human breast cancer cells with an $\mathrm{IC}_{50}$ of $0.01 \mu \mathrm{g} / \mathrm{ml}$ [30]. Recently, methyl nomilinate from citrus was shown to inhibit cell proliferation in the SW480 human colon cancer cell line through the modulation of G1 cell cycle regulators [31].

\section{CONCLUSION}

Phytochemical investigation of the EtOH extract of $C$. unshiu fruit led to the isolation and identification of nine compounds including one limonoid and eight flavonoids. AChE inhibition has been considered one of the promising temporary treatments for $\mathrm{AD}$. The inhibitory effect of these isolates on AChE activity was evaluated using a micro-well plate AChE inhibition assay, which indicated that the limonoid, methyl nomilinate (1), exhibited significant AChE inhibitory activity. This is the first study to identify the limonoid methyl nomilinate from $C$. unshiu fruit as a natural AChE inhibitor. Additionally, our findings suggest that $C$. unshiu fruit, one of the most popular fruits in Korea, can serve as a valuable natural, functional food source of therapeutic agents for AD.
Acknowledgements. This research was supported by the Basic Science Research Program through the National Research Foundation of Korea (NRF) funded by the Ministry of Science, ICT \& Future Planning (2015R1C1A1A02037383) and by the Ministry of Education (NRF-2012R1A5A2A28671860).

\section{REFERENCES}

[1] G. McKhann, D. Drachman, M. Folstein, R. Katzman, D. Price, E. M. Stadlan, Clinical diagnosis of Alzheimer's disease: report of the NINCDS-ADRDA Work Group under the auspices of Department of Health and Human Services Task Force on Alzheimer's Disease, Neurology 34(7), 939-944 (1984). DOI: 10.1212/WNL.34.7.939.

[2] D. J. Selkoe, P. J. Lansbury Jr., Basic Neurochemistry: Molecular, Cellular and Medical Aspects. sixth ed., Lippincott-Raven, Philadelphia, 1999.

[3] A. Enz, R. Amstutz, H. Boddeke, G. Gmelin, J. Malanowski, Brain selective inhibition of acetylcholinesterase: A novel approach to therapy for Alzheimer's disease, Prog. Brain Res. 98, 431-438 (1993). DOI: 10.1016/S0079-6123(08)62429-2.

[4] R. S. Shah, H. G. Lee, Z. Xiongwei, G. Perry, M. A. Smith, R. J. Castellani, Current approaches in the treatment of Alzheimer's disease, Biomed. Pharmacother. 62, 199-207 (2008). DOI: 10.1016/j.biopha.2008.02.005.

[5] K. Ingkaninan, P. Temkitthawon, K. Cheunchom, T. Yuyaem, W. Thongnoi, Screening for acetylcholinesterase inhibitory activity in plants used in Thai traditional rejuvenating and neurotonic remedies, J. Ethnopharmacol. 89, 261-264 (2003). DOI: 10.1016/j.jep.2003.08.008.

[6] E. Scarpini, P. Scheltens, H. Feldman, Treatment of Alzheimer's disease: current status and new perspectives, Lancet Neurol. 2(9), 539- 547 (2003). DOI: 10.1016/S1474-4422(03)00502-7.

[7] H. J. Eom, D. Lee, S. Lee, H. J. Noh, J. W. Hyun, P. H. Yi, K. S. Kang, K. H. Kim, Flavonoids and a limonoid from the fruits of Citrus unshiu and their biological activity, J. Agric. Food Chem. 64(38), 7171-7178 (2016). DOI: $10.1021 /$ acs.jafc.6b03465.

[8] C. I. Cheigh, E. Y. Chung, M. S. Chung, Enhanced extraction of flavonones hesperidin and narirutin from Citrus unshiu peel using subcritical water, J. Food Eng. 110, 472-477 (2012). DOI: 10.1016/j.jfoodeng.2011.12.019.

[9] S. Lee, J. Ra, J. Y. Song, C. Gwak, H. J. Kwon, S. V. Yim, S. P. Hong, J. Kim, K. H. Lee, J. J. Cho, Y. S. Park, C. S. Park, H. J. Ahn, Extracts from Citrus unshiu promote immune-mediated inhibition of tumor growth in a murin renal cell carcinoma model, J. Ethnopharmacol. 133, 973-979 (2011). DOI: 10.1016/j.jep.2010.07.018.

[10] M. Y. Choi, C. Chai, J. H. Park, J. Lim, J. Lee, S. W. Kwon, Effect storage period and heat treatment on phenolic compound composition in dried Citrus peels (Chenpi) and discrimination of chenpi with different storage periods through targeted metabolomics study using HPLC-DAD analysis, J. Pharm. Biomed. Anal. 54 (4), 638-645, (2011). DOI: 10.1016/j.jpba.2010.09.036.

[11] T. Inoue, S. Tsubaki, K. Ogawa, K. Onishi, J. Azuma, Isolation of hesperidin from peels of thinned Citrus un- 
shiu fruits by microwave-assisted extraction, Food Chem. 123, 542-547 (2010).

DOI: 10.1016/j.foodchem.2010.04.051.

[12] Y. Q. Ma, X. Q. Ye, Z. X, Fang, J. C. Chen, G. H. Xu, D. H. Liu, Phenolic compounds and antioxidant activity of extracts from ultrasonic treatment of satsuma mandarin (Citrus unshiu Marc.) peels, J. Agric. Food Chem. 56, 5682-5690 (2008). DOI: 10.1021/jf072474o.

[13] H. J. Noh, D. Hwang, E. S. Lee, J. W. Hyun, P. H. Yi, G. S. Kim, S. E. Lee, C. Pang, Y. J. Park, K. H. Chung, G. D. Kim, K. H. Kim, Anti-inflammatory activity of a new cyclic peptide, citrusin XI, isolated from the fruits of Citrus unshiu, J. Ethnopharmacol. 163, 106-112 (2015). DOI: 10.1016/j.jep.2015.01.024.

[14] J. P. Dzoyem, A. T. Tsamo, R. Melong, P. Mkounga, A. E. Nkengfack, L. J. McGaw, J. N. Eloff, Cytotoxicity, nitric oxide and acetylcholinesterase inhibitory activity of three limonoids isolated from Trichilia welwitschii (Meliaceae), Biol. Res. 48, 57 (2015). DOI: 10.1186/s40659-015-0049-0.

[15] R. D. Bennett, S. Hasegawa, Z. Herman, Glucosides of acidic limonoids in citrus, Phytochemistry 28 (10), 2777-2781 (1989). DOI: 10.1016/S0031-9422(00)98087-7.

[16] K. Machida, K. Osawa, On the flavonoid constituents from the peels of Citrus hassaku Hort. ex Tanaka, Chem. Pharm. Bull. 37(4), 1092-1094 (1989). DOI: $10.1248 / \mathrm{cpb} .37 .1092$.

[17] K. Kazuma, N. Noda, M. Suzuki, Malonylated flavonol glycosides from the petals of Clitoria ternatea, Phytochemistry 62 (2), 229-237 (2003). DOI: 10.1016/S0031-9422(02)00486-7.

[18] B. Bennini, A. J. Chulia, M. Kaouadji, F. Thomasson, Flavonoid glycosides from Erica cinera, Phytochemistry 31 (7), 2483-2486 (1992). DOI: $10.1016 / 0031-9422(92) 83305-I$.

[19] R. Liu, L. Kong, A. Li, A. Sun, Preparative isolation and purification of saponin and flavone glycoside compounds from Clinopodium chinense (Benth) O. Kuntze by high speed countercurrent chromatography, J. Liq. Chromatogr. Relat. Technol. 30 (4), 521-532 (2007). DOI: $10.1080 / 10826070601093846$.

[20] H. Kumamoto, Y. Matsubara, Y. Iizuka, K. Okamoto, K. Yokoi, Structures and hypotensive effect of flavonoid glycosides in unshiu peel. II, J. Jpn. Oil Chem. Soc. 35 (5), 379-381 (1986). DOI: 10.5650/jos1956.35.379.

[21] Y. Matsubara, H. Kumamoto, Y. Iizuka, T. Murakami, K. Okamoto, H. Miyake, K. Yokoi, Structure and hypotensive effect of flavonoid glycosides in Citrus unshiu peelings, Agric. Biol. Chem. 49 (4), 909-914 (1985). DOI: $10.1271 / \mathrm{bbb} 1961.49 .909$.
[22] G. Zhan, J. Zhou, R. Liu, T. Liu, G. Guo, J. Wang, M. Xiang, Y. Xue, Z. Luo, Y. Zhang, G. Yao, Galanthamine, plicamine, and secoplicamine alkaloids from zephyranthes candida and their anti-acetylcholinesterase and anti-inflammatory activities, J. Nat. Prod. 79 (4), 760-766 (2016). DOI: 10.1021/acs.jnatprod.5b00681.

[23] M. Samochocki, A. Höffle, A. Fehrenbacher, R. Jostock, J. Ludwig, C. Christner, M. Radina, M. Zerlin, C. Ullmer, E. F. R. Pereira, H. Lübbert, E. X. Albuquerque, A. Maelicke, Galantamine is an allosterically potentiating ligand of neuronal nicotinic but not of muscarinic acetylcholine receptors, J. Pharmacol. Exp. Ther. 305 (3), 1024-1036 (2003). DOI: 10.1124/jpet.102.045773.

[24] N. Li, Y. Liu, W. Li, L. Zhou, Q. Li, X. Wang, P. He, A UPLC/MS-based metabolomics investigation of the protective effect of ginsenosides $\operatorname{Rg} 1$ and $\mathrm{Rg} 2$ in mice with Alzheimer's disease, J. Ginseng Res. 40 (1), 9-17 (2016). DOI: 10.1016/j.jgr.2015.04.006.

[25] T. Xu, X. Shen, H. Yu, L. Sun, W. Lin, C. Zhang, Water-soluble ginseng oligosaccharides protect against scopolamine-induced cognitive impairment by functioning as an antineuroinflammatory agent, J. Ginseng Res. 40 (3), 211-219 (2016). DOI: 10.1016/j.jgr.2015.07.007.

[26] J. R. Bae, B. D. Lee, Function and dysfunction of leucine-rich repeat kinase 2 (LRRK2): Parkinson's disease and beyond. BMB Rep. 48 (5), 243-248 (2015). DOI: 10.5483/BMBRep.2015.48.5.032.

[27] J. Y. Sim, M. A. Kim, M. J. Kim, W. J. Chun, Y. S. Kwon, Acetylcholinesterase inhibitors from the stem of Zea mays, Nat. Prod. Sci. 20 (1), 13-16 (2014).

[28] A. Roy, S. Saraf, Limonoids: Overviews of significant bioactive triterpenes distributed in plants kingdom, Biol. Pharm. Bull. 29 (2), 191-201 (2006). DOI: $10.1248 / \mathrm{bpb} .29 .191$

[29] S. B. Liu, W. L. Mei, H. Q. Chen, Z. K. Guo, H. F. Dai, Z. N. Wang, Mexicanolide-type limonoids from the roots of Trichilia sinensis, Molecules 21 (9), 1152 (2016). DOI: 10.3390/molecules21091152.

[30] N. Guthrie, K. Morley, S. Hasegawa, G. D. Manners, T. Vandenberg, Inhibition of human breast cancer cells by citrus limonoids. ACS Symp. Ser. 758, 164-174 (2000). DOI: $10.1021 / \mathrm{bk}-2000-0758 . c h 012$.

[31] J. Kim, G. K. Jayaprakasha, A. Vikram, B. S. Patil, Methyl nomilinate from citrus can modulate cell cycle regulators to induce cytotoxicity in human colon cancer (SW480) cells in vitro. Toxicol. In Vitro 26 (7), 12161223 (2012). DOI: 10.1016/j.tiv.2012.06.005. 